\title{
Light dependent courtship behavior in Drosophila simulans and D. melanogaster
}

\author{
Michael P Shahandeh ${ }^{\text {Corresp., } 1}$, Cameryn Brock ${ }^{1}$, Thomas L Turner ${ }^{1}$ \\ ${ }^{1}$ Ecology, Evolution, and Marine Biology, University of California, Santa Barbara, Santa Barbara, California, United States \\ Corresponding Author: Michael P Shahandeh \\ Email address: mshahandeh@ucsb.edu
}

Differences in courtship signals and perception are well-known among Drosophila species. One such described difference is the dependency on light, and thus presumably vision, for copulation success. Many studies have described a difference in light-dependent copulation success between $D$. melanogaster and $D$. simulans, identifying $D$. simulans as a light-dependent species, and $D$. melanogaster as a light-independent one. However, many of these studies use assays of varying design and few strains to represent the entire species. Here, we attempt to better characterize this purported difference using 11 strains of each species, paired by collection location, in behavioral assays conducted at two different exposure times. We show that, while there is a species-wide difference in magnitude of light-dependent copulation success, $D$. melanogaster copulation success is, on average, still impaired in the dark at both exposure times we measured. Additionally, there is significant variation in strain-specific ability to copulate in the dark in both species across two different exposure times. We find that this variation correlates strongly with longitude in $D$. melanogaster, but not in $D$. simulans. We hypothesize that differences in species history and demography may explain behavioral variation. Finally, we use courtship assays to show that light-dependent copulation success in one $D$. simulans strain is driven in part by both males and females. We discuss potential differences in courtship signals and/or signal importance between these species and potential for further comparative studies for functional characterization. 
2

3 Michael P. Shahandeh ${ }^{1}$, Cameryn Brock ${ }^{1}$, and Thomas L. Turner ${ }^{1}$

$4 \quad{ }^{1}$ Ecology, Evolution, and Marine Biology, University of California, Santa Barbara, Santa

5 Barbara, California, United States

6 Corresponding author:

7 Michael P. Shahandeh

8 mshahandeh@ucsb.edu 


\section{Abstract}

10 Differences in courtship signals and perception are well-known among Drosophila species. One

11 such described difference is the dependency on light, and thus presumably vision, for copulation

12 success. Many studies have described a difference in light-dependent copulation success between

13 D. melanogaster and $D$. simulans, identifying $D$. simulans as a light-dependent species, and $D$.

14 melanogaster as a light-independent one. However, many of these studies use assays of varying

15 design and few strains to represent the entire species. Here, we attempt to better characterize this

16 purported difference using 11 strains of each species, paired by collection location, in behavioral

17 assays conducted at two different exposure times. We show that, while there is a species-wide

18 difference in magnitude of light-dependent copulation success, D. melanogaster copulation

19 success is, on average, still impaired in the dark at both exposure times we measured.

20 Additionally, there is significant variation in strain-specific ability to copulate in the dark in both

21 species across two different exposure times. We find that this variation correlates strongly with

22 longitude in D. melanogaster, but not in D. simulans. We hypothesize that differences in species

23 history and demography may explain behavioral variation. Finally, we use courtship assays to

24 show that light-dependent copulation success in one $D$. simulans strain is driven in part by both

25 males and females. We discuss potential differences in courtship signals and/or signal

26 importance between these species and potential for further comparative studies for functional

27 characterization.

28

29 Keywords: Drosophila, courtship, signals, perception, vision 
30

31

32

33

34

35

36

37

38

39

40

41

42

43

44

45

46

47

48

49

50

51

52

53

54

55

56

57

58

59

60

\section{Introduction}

Courtship in Drosophila is a multimodal form of communication, involving chemosensory, auditory, tactile, and visual signals (Greenspan \& Ferveur, 2000). Often, male signals are more conspicuous and easily observed, and thus are more widely studied; for example, males of many species produce a courtship song by extending and vibrating a single wing (Spieth, 1952). The resulting song can be recorded and separated into discrete parts, such as pulse and sine song (Schilcher, 1976), and quantified using metrics like inter-pulse interval and pulse duration (Kyriacou \& Hall, 1982). These metrics show clear signs of species specificity that are important to conspecific reproductive success (Kyriacou \& Hall, 1982; Spieth, 1974). Another signal that can be easily quantified, and has been extensively studied, is variation in chemotactic pheromones (Cobb \& Jallon, 1990a; Jallon \& David, 1987; Pardy et al., 2018; Pischedda et al., 2014). These pheromones, present on the fly cuticle, sometimes act as sex- and species-specific identifiers that stimulate courtship among conspecific pairings, but suppress courtship between heterospecific pairs in some species (Billeter et al., 2009; Manning, 1959b; Savarit et al., 1999; Shahandeh et al., 2018, Shahandeh et al., 2020).

In the case of visual signals, for Drosophila melanogaster, visual perception of a moving courtship target is necessary for males to initiate and maintain courtship (Agrawal et al., 2014;

Cook, 1980), with males preferring to initiate courtship towards moving targets over stationary ones (Tompkins et al., 1982). D. melanogaster males prefer larger females (Byrne \& Rice, 2006; Edward \& Chapman, 2012), though it remains to be determined if this choice is driven by visual perception or some other cryptic correlate of female quality. Given that these prior results highlight the potential importance of visual signaling in courtship, it is noteworthy that $D$. melanogaster is said to copulate successfully independent of light (Manning, 1959a; Spieth \& Hsu, 1950). Indeed, for many other Drosophila species, copulation success is relatively light dependent (Ewing, 1983; Grossfield, 1971; Spieth, 1974). However, the specific visual signals that make courtship light dependent for other species remain relatively unclear, with a few notable exceptions where males or females have evolved an additional postural display whereby they presumably send visual signals using specific, repeated, positions or movements (Brown, 1965; Ewing, 1983).

For the commonly studied cosmopolitan species, $D$. simulans, no such postural behavior has been described. Nonetheless, this species is said to differ largely from D. melanogaster in 
61 their light-dependent copulation success (Grossfield, 1971; Manning, 1959a). Specifically, D.

62 melanogaster copulates successfully independent of light, while $D$. simulans copulates

63 significantly less in the dark (Manning, 1959a; Spieth \& Hsu, 1950). The ubiquity of this

64 purported difference is debatable, however, as genetically blinded D. melanogaster do not

65 copulate as successfully as wild-type males when kept in bright light (Tompkins et al., 1982).

66

67

68

69

70

71

72

73

74

75

76

77

78

79

80

81

82

83

84

85

86

87

88

89

90

91
Reported differences in Drosophila light-dependent copulation behavior may be a result of strain-specific behavior or may reflect experimentally induced variation. Indeed, studies often use just one or two strains as a representative of a species and conduct assays of variable lengths, ranging from minutes to a week, and designs, ranging from individual pairs of flies to large groups that are blinded or compared under varying light regimes (Cobb \& Ferveur, 1995; Giglio \& Dyer, 2013; Gleason et al., 2012; Grossfield, 1971; Manning, 1959a; Spieth et al., 1950, Tompkins et al., 1982).

In the present study, we seek to more accurately quantify the level of light dependency for these two sister species of Drosophila: D. melanogaster and D. simulans. To do so, we measure light dependent copulation success at two exposure times for 11 strains of $D$. melanogaster and 11 strains of $D$. simulans collected from paired locations around the globe. By doing so, we are able to quantify species differences in light dependent copulation behavior as well as assess intraspecific variation and time-dependency. Further, there is some evidence that light-dependent copulation success is inversely correlated with ecological generality (Grossfield, 1971). Using our D. melanogaster and D. simulans strains, we also test for correlations among behavior and geographic variables to gain insight into potential factors underlying global behavioral variation. Finally, we use the most extreme lines from either side of the behavioral spectrum for each species in courtship observation to begin to understand the mechanistic causes of light-dependent behavior.

\section{Materials and Methods}

\section{Fly strains and maintenance}

We selected 11 wild-type D. melanogaster and 11 wild-type D. simulans strains (Table 1) from the National Drosophila Species Stock center that were collected across 6 continents. These 22 strains constitute 11 pairs of $D$. simulans and D. melanogaster strains that were collected at approximately equal latitudes and longitudes. Whenever possible, we chose strains that were

Peer) reviewing PDF | (2019:11:43015:3:0:NEW 12 Jun 2020) 
92 collected from the same location at the same time.

93 We maintained each strain on non-overlapping, alternating 2-week life cycles. We reared

94 all strains on a standard cornmeal-yeast-molasses medium in $25 \mathrm{~mm}$ vials at $25^{\circ} \mathrm{C}$ and $\sim 50 \%$

95 humidity under a 12:12 hour light/dark cycle. At the beginning of each cycle, we transferred

96 roughly 20-30 adult flies to a culture vial with fresh media. We allowed the flies to oviposit for

9748 hours before transferring them to a second, collection vial with fresh media, where flies were

98 allowed to oviposit for an additional 24 hours before being discarded. We repeated this process

99 every fourteen days using offspring from the culture vials to maintain each strain. For use in

100 experiments, we collected male and female offspring as virgins from the collection vials 4-5

101 hours following "lights-on" under light $\mathrm{CO}_{2}$ anesthesia 11 days after oviposition. For all

102 experiments described below, we aged males and females separately in holding vials with fresh

103 food media at a density of 10 flies for 3-4 days before each assay. We aged flies in groups prior

104 to assay, because flies held in isolation display increased aggressive behaviors we were

105 concerned would affect copulation success, skewing our data (Hoffmann, 1990).

106

107 Light dependent copulation success assay

108 To measure each strain's ability to successfully copulate independent of light, we measured 109 copulation success in a normal light (control) treatment, and in an entirely dark (experimental)

110 treatment, side-by-side on the same day. On the morning of each assay, immediately following

111 "lights-on", we aspirated a single virgin male and female into a $20 \mathrm{~mm}$ vial with fresh food

112 media sealed with a foam plug. We chose to use single pairs, as there is some evidence from

113 other Drosophila that males approach females sequentially in the wild, and females that are

114 approached singly are more likely to copulate (Noor \& Ortiz-Barrientos, 2006). We assayed flies

115 in vials with food media because adult Drosophila are most likely to encounter mates on or near

116 a food substrate (Soto-Yéber et al., 2018). We held these vials at $25^{\circ} \mathrm{C}$ and $\sim 50 \%$ humidity for

117 either 2 or 6 hours in an incubator illuminated with a Phillips "Cool white" 32-watt fluorescent

118 light bulb,

119 or in the same incubator sealed in a light-proof box (experimental). We chose these time-points,

120 rather than a day or week-long assay, because they represent a short, more realistic exposure

121 time for flies in the wild, and a longer exposure time with which we could assess time

122 dependency. At the end of the assay, we used an aspirator to remove the male from the vial so no

Peer] reviewing PDF | (2019:11:43015:3:0:NEW 12 Jun 2020) 
123 post-assay copulation could occur. We then held females in vials at $25{ }^{\circ} \mathrm{C}$ and $\sim 50 \%$ humidity in 124 a 12h:12h light/dark cycle to oviposit for 7 days. On day seven, we checked each vial for the 125 presence of larvae or early stage pupae, indicating whether insemination successfully occurred 126 during the assay time. We collected all data on a weekly basis over the course of 6 weeks.

127 For each of the 11 D. melanogaster and D. simulans strains, we observed 25-31 pairings 128 in the control and experimental treatments for each exposure time ( 2 or 6 hours). For each strain, 129 we calculated copulation success using the proportion of vials that produced offspring as a proxy 130 for the proportion of vials where successful copulation occurred. We used Fisher's exact tests to 131 compare copulation success between control and experimental treatments for each strain at each 132 exposure time, followed by post-hoc correction for multiple comparisons (Holm, 1979). We 133 tested for a species-wide difference between light/dark treatments at each exposure time using 134 paired t-tests or paired Wilcoxon rank-sum tests when the data did not fit a normal distribution.

135 We next calculated relative dark copulation success for each exposure time as the percent 136 of successful copulations in the dark treatment divided by the proportion of successful 137 copulations for the same strain in the light treatment (\% dark / \% light). We compared relative 138 dark copulation success between the 2-hour and 6-hour exposure times within species, and for 139 each exposure time between species, using paired t-tests. We also tested for a correlation 140 between relative dark copulation success at each exposure time and three collection variables: 141 longitude, the absolute value of latitude (i.e. distance from the equator), and collection date 142 (when available). We used Pearson's correlation test when the data fit a normal distribution, and 143 Spearman's rank correlation when the data was not normally distributed.

144

\section{Courtship assays}

146 To test if males from $D$. melanogaster and $D$. simulans strains with relatively light-dependent 147 and light-independent copulation success actively court females in the dark, we conducted 148 courtship assays under two treatments. For each treatment, we gently aspirated single virgin 149 males into vials containing a thin layer of food media 24 hours prior to the assay. The morning of 150 the assay, 1-2 hours following "lights on," we aspirated a single female into the vial and pushed 151 a foam plug down into the vial until it was just $1-2 \mathrm{~cm}$ from the food surface. Because these flies 152 were held in a much smaller space, they were forced to interact even when held in the dark, so 153 we observed courtship for just 30 minutes to avoid observer fatigue. We scored each minute for 
154 one of three easily distinguished courtship behaviors: (1) singing (single-wing extension and 155 vibration), (2) attempted copulation, and (3) successful copulation. For males exhibiting multiple 156 behaviors within a minute, we scored each pairing once per minute per behavior. As a control, 157 we observed male and female pairings under bright light. As an experimental treatment, we 158 observed male and female pairings in a dark room illuminated solely with red light because the 159 Drosophila compound eye is insensitive to red wavelengths of light (Salcedo et al., 1999). 160 We selected the single most light-independent and light-dependent $D$. simulans strains 161 (MAL261 and SA169, respectively) and D. melanogaster strains (M-BAZ15 and M-NG120, 162 respectively) from the two-hour exposure period for courtship observation. First, for all four 163 strains, we observed males with females of their own strain. For the $D$. simulans strains, we also 164 observed males with females collected from the opposing strain because SA169 displayed 165 significantly less frequent courtship towards SA169 females when observed in the dark (see 166 results). In either treatment, we considered any male that spent more than $10 \%$ of the assay time 167 exhibiting any courtship behavior as successfully courting. We compared the proportion of males 168 that courted females in the light and in the dark using Fisher's exact tests followed with post-hoc 169 correction for multiple comparisons (Holm, 1979). For males that displayed courtship, we also calculated courtship latency (the time from the start of assay until the male initiates courtship) and courtship effort (the total proportion of time a male spends courting during the 30-minute assay). If a pair successfully copulated, we calculated courtship effort as the percent of time a male spent courting from start of assay until copulation. We did not apply the same $10 \%$ cut-off for courtship latency and effort, as we did for the proportion of males that courted, due to exceedingly low sample sizes. We compared courtship latencies and courtship efforts between pairings using Wilcoxon rank-sum tests followed by post-hoc correction for multiple comparisons (Holm, 1979).

\section{Results}

\section{Light dependent copulation success}

\section{D. simulans}

182 For D. simulans, copulation is much more successful in the light than in the dark when males and 183 females were held together for 2 hours (paired t-test, $\mathrm{p}<0.0001$, Fig. 1A). On average, $D$. 
185 when given 2 hours. Each of the 11 strains had decreased copulation success in the dark, and 8 of 18611 strains were statistically significant after correcting for multiple comparisons (Fig. 1A, Table 187 S1). The species-level pattern is still detectable when males and females are held together for 6 188 hours, but far less significant (paired Wilcoxon, $\mathrm{p}<0.05$, Fig. 1B). Overall, D. simulans strains 189 were $77.36 \%$ as successful at copulating in the dark as they were when held in the light for 6 190 hours. This marked improvement appears to be driven by six lines (NUE006, MAL261, 191 GUY001, FLO166, PER005, and MAD196), which copulate approximately equally as 192 successfully in the light as they do in the dark when given increased time (all $\mathrm{p}=1$, Fig. 1B, 193 Table S1). Still, the remaining lines displayed reduced copulation successes in the dark 194 compared to the light treatment, with 3 remaining significantly lower following correction for 195 multiple comparisons (Fig. 1B, Table S1). When we compared the relative dark copulation 196 success for our $D$. simulans strains across the two exposure times, we found a significant 197 difference between the 2 and 6-hour treatments (paired Wilcoxon $\mathrm{p}<0.001$ ), with strains 198 showing decreased light dependency for copulation at the 6-hour exposure time (Fig. 1C). 199 Overall, the proportion of flies that successfully copulated increased by 0.36 , on average, when 200 given extra time.

201

202 D. melanogaster

203 We also found that copulation is more successful in the light for D. melanogaster when pairs 204 were given 2 hours to mate (paired t-test $\mathrm{p}<0.001$, Fig. 2A). On average, D. melanogaster was $20565.54 \%$ as effective at mating in the dark as they were in the light when given 2 hours, with 6 of 20611 strains significantly worse in the dark after correcting for multiple comparisons (Fig. 2A, 207 Table S1). Like with D. simulans, we were still able to detect an overall effect of light vs. dark 208 treatments at 6 hours, albeit less significantly (paired Wilcoxon p < 0.01, Fig. 2B, Table S1). 209 While just two lines individually copulated significantly less at this exposure time (M-MAD125 210 and M-NG120), all lines showed a reduced proportion of copulating pairs in the dark relative to 211 the light. Overall, the difference between treatments at 6 hours was smaller, with strains 212 copulating $80.24 \%$ as successfully in the dark as they did in the light. Again, we find that $D$. 213 melanogaster males show reduced light-dependent copulation behavior at 6 hours relative to 2 214 hours (paired Wilcoxon $\mathrm{p}<0.01$, Fig. 2C). Overall, the proportion of flies that successfully copulated increased by 0.15 , on average, when given extra time. 


\section{Comparing D. simulans and D. melanogaster}

218 When we compare relative copulation success in the dark between $D$. simulans and $D$.

219

220

221

222

223

224

225

226

227

228

229

230

231

232

233

234

235

236

237

238

239

240

241

\section{2}

243

244

245

246 melanogaster, we see a significant difference at the 2-hour exposure time (paired t-test $\mathrm{p}<0.05$ ). Specifically, the relative copulation success in the dark for D. simulans (41.77\%) is significantly lower than that of $D$. melanogaster (65.54\%). Contrastingly, we do not find a significant difference at the 6-hour exposure time (paired Wilcoxon $\mathrm{p}=0.5988$ ). The loss of the effect is driven by $D$. simulans strains improving their relative copulation success in the dark significantly when given more time $(77.36 \%)$, compared to a more minor improvement by D. melanogaster (80.24\%). We also found no correlation between the light-dependent copulation behavior of $D$. simulans strains and the D. melanogaster strains collected from the same (or similar) geographic location at 2 hours (Pearson's $r=0.1679, p=0.6217$ ) or 6 hours (Spearman's rho $=0.2115, \mathrm{p}=$ $0.5324)$.

\section{Correlations of light-dependent copulation success}

Because we found the largest effect of light-dependent copulation success at the 2-hour exposure time, we used the relative copulation success in the dark of our 11 D. melanogaster and $D$. simulans strains at 2 hours to test for a correlation with other variables: aspects of collection location and date. For our D. simulans strains, we found no correlation between behavior and longitude (Pearson's $r=-0.2787, p=1$, Fig. 3B), distance from the equator (Pearson's $r=-$ $0.2579, p=1$, Fig. S1A), or collection date (Spearman's rho $=0.2152, p=1$, Fig. S2A). For our D. melanogaster strains, we found no correlation between behavior and distance from the equator (Pearson's $r=0.2490, p=1$, Fig. S1B) or collection date (Pearson's $r=0.0700, p=1$, Fig. S2B). We did, however, detect a significant correlation between light-dependent copulation success at 2 hours and longitude (Pearson's $r=-0.8617, \mathrm{p}<0.01$, Fig. 3C).

\section{Light-dependent courtship behavior}

We wanted to know if light-dependent copulation behavior was mediated by male or female behavior. To test for differences in courtship behavior, we observed the courtship of two $D$. melanogaster strains under bright light and in darkness (Fig. 4A, Table S2A). For the strain we identified as relatively light-independent using our copulation assay, M-BRAZ15, we found that 
247 males court females at high frequencies in both treatments. Specifically, 100\% of M-BRAZ15

248 males courted their own females under bright light, while $80 \%$ displayed courtship towards

249 females when observed in the dark. Strain M-NG120, which displayed the most light-dependent

250 copulation in our assay, courted females $50 \%$ of the time under bright light, and $30 \%$ of the time

251 in the dark. While each strain showed a $20 \%$ decrease in overall courtship, the difference was not

252 significant in either case ( $\mathrm{p}=0.9474$ for both). Additionally, we detected no significant

253 differences between courtship latency or effort for pairings observed in the light compared to

254 those in the dark, however sample sizes are quite small, as not all males displayed courtship

255 (Table S3).

256 We also observed the courtship behavior of two D. simulans strains under the same 257 conditions (Fig. 4B, Table S2A). MAL261, which copulated successfully independent of light,

258 displayed high courtship towards their own females in both scenarios; $100 \%$ of males courted in

259 the light, while $71.4 \%$ displayed courtship in the dark $(\mathrm{p}=0.4909)$. For SA169 males, which

260 showed the strongest signal of light-dependent copulation success in our mating trials, we

261 observed a significant difference in the proportion of males that courted under bright light,

$262100 \%$, compared to in the dark, $14.3 \%(\mathrm{p}<0.05)$. For both MAL261 and SA169 we did not

263 detect significant differences in courtship latency or effort when courting in the dark relative to

264 the light (Table S3). To identify whether the difference in the proportion of courtship we

265 observed is driven by male or female behavior, we observed MAL261 males with SA169

266 females and SA169 males with MAL261 females under the same conditions (Fig. 4C, Table

267 S2B). We found that $75 \%$ of MAL261 males still court SA169 females in the dark, compared to

$26866.7 \%$ in the light $(\mathrm{p}=1)$. Interestingly, we also found that SA169 males court MAL261 equally

269 as well in the dark as they do in the light $(100 \%$ for both, $p=1)$. Again, there were no detectable

270 differences in courtship latency or effort at these small sample sizes (Table S3).

271

272 Discussion

273 A species difference in light-dependent copulation behavior

274 In some respects, our findings confirm a previously described species difference in light275 dependent copulation behavior (Grossfield, 1971; Spieth, 1974). Specifically, D. melanogaster

276 strains had, on average, greater copulation success in the dark than D. simulans strains when

277 assayed for 2 hours. However, this difference disappears when strains of each species are given 4 
278 more hours. While both species show improvement in copulation success in the dark when given

279 increased time, the loss of a species difference at 6 hours is driven by a significant improvement

280 in ability to copulate in the dark among D. simulans strains relative to D. melanogaster strains.

281 Interestingly, the overall improvement is largely driven by 6 D. simulans strains (Fig. 1), that

282 copulate equally as well in the dark as in the light when given 6 hours.

283 In other measures, our results refute some of the species-wide conclusions made by

284 previous studies of light-dependent copulation success, and address some of the inconsistencies

285 among previously published results. First, we show that D. melanogaster strains, as a whole, do

286 not copulate successfully independent of light. At both the 2 and 6-hour exposure times, we

287 detect a significant difference in copulation success between our light and dark treatments.

288 Individually, in both treatments, each strain has a higher copulation success in the light compared

289 to the dark ( 6 strains and 2 strains significantly so at 2 and 6 hours respectively). This is in

290 contrast to $D$. simulans, where a handful of strains show relatively unchanged, if not slightly

291 increased, copulation success in the dark relative to the light at the 6-hour exposure time.

292 Overall, while these data do demonstrate a difference between species in light-dependent

293 copulation behavior, they also highlight an important role of both genetic variation and assay

294 time in detecting this species difference. Our data show that a high level of intraspecific variation

295 for both species creates largely overlapping distributions of behavior that are greatly affected by

296 exposure time. Had we chosen fewer strains or sampled at a single exposure time, our

297 conclusions may have been very different.

298

299 Environmental correlates of light-dependent copulation behavior

300 We observed substantial variation in light-dependent copulation behavior among our $D$.

301 simulans and D. melanogaster strains. Previous results have suggested a role of ecological

302 generality in dark copulation success (Grossfield, 1971); species that occupy greater geographic

303 range tend to have increased ability to copulate successfully in the dark. However, both $D$.

304 simulans and D. melanogaster are near cosmopolitan human commensal species that overlap

305 nearly entirely in their geographic ranges (Kliman et al., 2000). To attempt to identify other

306 potential causes of the species difference we detected at the 2-hour exposure time, we tested for

307 correlations between light-dependent copulation success and other aspects of strain collection

308 (latitude, longitude, and collection date). We found no correlation between collection date and

Peer] reviewing PDF | (2019:11:43015:3:0:NEW 12 Jun 2020) 
309 behavior for either D. melanogaster or D. simulans, which reduces (but does not conclusively

310 eliminate) the likelihood that variation in light-dependent copulation behavior is a product of

311 laboratory adaptation. For $D$. simulans, light-dependent copulation behavior did not correlate

312 with either longitude or distance from the equator. Further studies including more strains would

313 have increased power to detect less significant correlations, however. For D. melanogaster,

314 behavioral variation correlated strongly with longitude at the strain's collection site (Fig. 3C).

315 This correlation is unlikely to be a result of differences in habitat, as longitude does not correlate

316 strongly with measures of environment. Instead, this correlation might reflect the demographic

317 history of D. melanogaster. If so, the lack of correlation between variation in D. simulans

318 behavior and longitude potentially reflects differences in species demography.

D. melanogaster originated in sub-Saharan Africa, eventually expanding out of Africa

320 and colonizing the rest of the world, first colonizing Eurasia (Li \& Stephan, 2006). Much later,

321 an admixed American population was established, likely during the modern colonization of the

322 Americas (<500 years ago) (Duchen et al., 2013). Australia's population is similarly admixed

323 and likely very recently colonized with modern sea travel (Arguello et al., 2019). Importantly,

324 there is still significant gene flow between populations (Arguello et al., 2019). Our data

325 potentially mirror these two recent independent trans-oceanic colonization events. American

326 populations show the highest level of light-independent copulation success, while

327 Australian/south east Asian populations display the lowest. The African and European

328 populations fall in the middle (for both behavior and longitude). If we consider the African

329 phenotypes ancestral, then there appears to be little change with the colonization of Europe, but

330 dramatic shifts in behavior with opposing valence for the colonization of the Americas and

331 Oceania. Whether these differences are due to founder effects or divergent selection remains an

332 open question. In contrast, D. simulans, which originated in East Africa or Madagascar, spread

333 across the globe much more recently (Dean \& Ballard, 2004), and globally distributed samples

334 show significantly less population structure (Irvin et al., 1998) and clinal variation (Machado et

335 al., 2016) than D. melanogaster. Similarly, there is no discernable structure to light-dependent

336 copulation success in our data. The effects of species demography on light-dependent copulation

337 success are hypotheses that still require explicit testing, however. We cannot make strong

338 conclusions regarding these effects with the data we have presented here. 
340 Courtship behavior differs between relatively light-dependent and light-independent 341 strains

342 To begin to identify the mechanistic drivers of differences in light-dependent copulation 343 behavior, we selected the most light-dependent and light-independent strains from each species 344 to compare male courtship rates in both the light and the dark. For D. melanogaster, we found 345 that both the light-independent (M-BRAZ15) and light-dependent (MNG120) strains courted at 346 statistically indistinguishable rates in both treatments. Although, M-NG120 showed reduced 347 courtship overall. This relative reduction in courtship is congruent with the result of our mating 348 assay, where M-NG120 showed the lowest copulation success in both the light and the dark, 349 indicating this pattern is more likely a result of differences in male courtship vigor. These results 350 imply that for these strains of D. melanogaster, reduced copulation success may depend partially 351 on a male's ability to locate females in the dark. Partly supporting this hypothesis, recent work 352 has shown that other Drosophila species display increased courtship latency in the absence of 353 visual cues (Roy \& Gleason, 2019). We did not detect significant differences in courtship 354 latency, but further observation at larger sample sizes may find a similar trend. Differences in 355 ability to locate females in the dark may be driven by a unique male scanning behavior described 356 among dark-courting D. melanogaster strains, presumably used to locate females without visual 357 input (Krstic et al., 2009). D. melanogaster males also depend on olfactory cues and female 358 movement (and the resulting sound/vibration) to initiate courtship with females in the dark 359 (Ejima \& Griffith, 2008; Stockinger et al., 2005). Thus, in the absence of visual detection of 360 movement, D. melanogaster males can rely on another sensory modalities to identify the 361 presence of courtship targets and adopt a different strategy to locate them in the dark. The 362 variation we observed among strains also potentially reflects variations in male's ability to locate 363 females, or variation in female signals that males use to locate females in the dark (Trajković et 364 al., 2017).

$365 \quad$ For D. simulans, we found that the light-independent strain (MAL261) courted females 366 with high frequency in the light, and somewhat reduced, although statistically insignificantly, 367 frequency in the dark. For the light-dependent D. simulans strain (SA169), we found that males 368 courted females at high frequencies in the light, but at significantly lower frequencies in the dark 369 (Fig. 4B). Thus, SA169 males are less willing or able to court SA169 females in the dark than 370 MAL261 males are able to court MAL261 females, despite having equal courtship in the light. 
371 From these results, it is unclear if the difference in the amount of courtship in the dark is driven

372 by males or females. It is possible that SA169 males cannot identify potential courtship targets

373 because of the lack of a perceivable moving courtship target. It could be equally likely that

374 SA169 female signals are missing or become indiscernible in the dark, leading to a reduction in 375 male courtship.

376 To test if the loss of SA169 male courtship is driven by female signals, we swapped

377 female types for our light-dependent and light-independent strains. We observed SA169 males

378 with MAL261 females, and vice versa. MAL261 males were equally able to court SA169

379 females in the light and in the dark. Surprisingly, we found the same pattern for SA169 males:

380 they court MAL261 females $100 \%$ of the time in both the light and in the dark. So, SA169 males

381 can locate and court females in the dark, but do not do so when those females are also SA169. In

382 contrast, MAL261 males will court either female in the dark. These results indicate that there is

383 both a difference in male strains' ability/willingness to court and in female strains' attractiveness

384 in the dark. It is possible that these differences are the result of differences in female activity or

385 male olfactory or vibratory/acoustic perception ability in the dark, but we do not know if $D$.

386 simulans males rely on olfactory, auditory, or vibratory cues to identify females in the dark in the

387 same way that D. melanogaster males do (Ejima et al., 2008). Ultimately, these results highlight

388 the complex coordination of signals and receivers that underlies Drosophila courtship. They also

389 suggest that the variation we observe among lines, potentially in both species, can reflect

390 variation in male behavior, female behavior, or both. More careful observation of a greater

391 number of flies and strains (and strain combinations) is necessary to understand the contributions

392 of males and females

393

394 The role of sensory perception in $D$. simulans light-independent courtship behavior

395 The above results highlight an important, yet unknown aspect of male mate choice in $D$.

396 simulans. While we know quite a bit about the signals that males send to females during

397 courtship (Greenspan et al., 2000), it remains unclear what signals D. simulans males use to

398 discriminate between males and females. Unlike its sister species, D. melanogaster, D. simulans

399 males and females express the same primary chemotactic pheromone, 7-tricosene (7T) (Cobb \&

400 Jallon, 1990b). It is possible that males discriminate between sexes using a difference in 7T

401 abundance, a difference in one of the many low-abundance cuticular hydrocarbons (CHCs) 
402 (Pardy et al., 2018), or the male-specific expression of cis-vaccenyl acetate (Jallon, 1984). While 403 D. simulans males and females can differ quantitatively in CHCs, this only seems to be the case 404 in some strains (Sharma et al., 2012). Additionally, the perception of these chemotactic signals 405 is light-independent, and are unlikely to explain the species-wide reduction in dark copulation 406 success anyway. Our results suggest that male courtship initiation in D. simulans may partially 407 depend on a female visual signal. They also suggest that strains can vary in the importance of 408 visual perception to initiate male courtship, which might reflect variation in ability to rely on 409 other sensory modalities to identify female courtship targets.

410

\section{Conclusions}

The above conclusions highlight two potential areas of further investigation. First, for

413 males that show higher light-dependent copulation success, what are the visual signals necessary

414 for courtship initiation? Second, for males that show relatively light-independent copulation 415 success, what other signals allow them to successfully initiate courtship in the dark? In either 416 case, the role of vision needs to be further examined in combination with the other senses in a 417 larger comparative framework to understand how sensory modalities interact to determine 418 variation male courtship behavior both within and between species. Should visual signals prove 419 important to D. simulans male mate discrimination, there is ample opportunity for a more precise 420 characterization of the neural substrate of signal hierarchies in D. simulans courtship behavior. In 421 D. melanogaster, sensory receptors (Ahmed et al., 2019; Ejima et al., 2008; Göpfert \& Robert, 422 2003; Montell, 2009) and specific neurons necessary to detect a variety of courtship signals (Lu 423 et al., 2014; Pan et al., 2012; Seeholzer et al., 2018; Starostina et al., 2012; Thistle et al., 2012; 424 Toda et al., 2012) and produce male courtship behavior (Kohatsu et al., 2011; von Philipsborn et 425 al., 2011) have been identified. Our results highlight that D. simulans may rely on different (or 426 differentially weighted) female signals. A comparative analysis of the molecular underpinnings 427 of signal perception in these species will help to identify differences in signal perception or 428 signal hierarchies.

429

430 Acknowledgements 
431 We are grateful to Dr. William R. Rice for the casual laboratory observation that inspired this

432 project.

433

434

\section{Author contributions}

435

436

437

438

439

440

441

442

443

444

445

446

447

448

449

450

451

452

453

454

455

456

457

458

459

460

461

462

463

464

465

466
TLT, MPS, and CB conceived of the study. MPS and CB collected and analyzed the data. MPS drafted the manuscript; CB and TLT revised it. All authors gave final approval for publication and agree to be held accountable for the work performed therein.

\section{Funding}

This work was supported by the NIH (R01 GM098614).

\section{References}

Agrawal, S., Safarik, S., \& Dickinson, M. (2014). The relative roles of vision and chemosensation in mate recognition of Drosophila melanogaster. Journal of Experimental Biology, 217(15), 2796-2805.

Ahmed, O. M., Avila-Herrera, A., Tun, K. M., Serpa, P. H., Peng, J., Parthasarathy, S., Knapp, J. M., Stern, D. L., Davis, G. W., Pollard, K. S., \& Shah, N. M. (2019). Evolution of Mechanisms that Control Mating in Drosophila Males. Cell Reports, 27(9), 2527-2536.e4.

Arguello, J. R., Laurent, S., Clark, A. G., \& Gaut, B. (2019). Demographic History of the Human Commensal Drosophila melanogaster. Genome Biology and Evolution, 11(3), 844-854.

Billeter, J.-C., Atallah, J., Krupp, J. J., Millar, J. G., \& Levine, J. D. (2009). Specialized cells tag sexual and species identity in Drosophila melanogaster. Nature, 461(7266), 987-991.

Brown, R. G. (1965). Courtship behaviour in the Drosophila obscura group. II. Comparative studies. Behaviour, 25(3), 281-323.

Byrne, P. G., \& Rice, W. R. (2006). Evidence for adaptive male mate choice in the fruit fly Drosophila melanogaster. Proceedings of the Royal Society B: Biological Sciences, 273(1589), 917-922.

Cobb, M., \& Ferveur, J.F. (1995). Evolution and genetic control of mate recognition and stimulation in Drosophila. Behavioral Processes, 35, 35-54.

Cobb, M., \& Jallon, J. M. (1990a). Pheromones, mate recognition and courtship stimulation in the Drosophila melanogaster species sub-group. Animal Behaviour, 39(6), 1058-1067.

Cobb, M., \& Jallon, J. M. (1990b). Pheromones, mate recognition and courtship stimulation in the Drosophila melanogaster species sub-group. Animal Behaviour, 39(6), 1058-1067.

Cook, R. (1980). The extent of visual control in the courtship tracking of D. melanogaster. Biological Cybernetics, 37(1), 41-51.

Dean, M. D., \& Ballard, J. W. O. (2004). Linking phylogenetics with population genetics to 
467

468

469

470

471

472

473

474

475

476

477

478

479

480

481

482

483

484

485

486

487

488

489

490

491

492

493

494

495

496

497

498

499

500

501

502

503

504

505

506

507

508

509

510

511

512

reconstruct the geographic origin of a species. Molecular Phylogenetics and Evolution, 32(3), 998-1009.

Duchen, P., Zivkovic, D., Hutter, S., Stephan, W., \& Laurent, S. (2013). Demographic inference reveals African and European admixture in the North American Drosophila melanogaster population. Genetics, 193(1), 291-301.

Edward, D. A., \& Chapman, T. (2012). Measuring the fitness benefits of male mate choice in Drosophila melanogaster. Evolution, 66(8), 2646-2653.

Ejima, A., \& Griffith, L. C. (2008). Courtship initiation is stimulated by acoustic signals in Drosophila melanogaster. PLoS ONE, 3(9), e3246.

Ewing, A. W. (1983). Functional aspects of Drosophila courtship. Biological Reviews, 58(2), 275-292.

Giglio, E. M., \& Dyer, K. A. (2013). Divergence of premating behaviors in the closely related species Drosophila subquinaria and D. recens. Ecology and Evolution, 3, 365-374.

Gleason, J. M., Pierce, A. A., Vezeau, A. L., \& Goodman, S. F. (2012). Different sensory modalities are required for successful courtship in two species of the Drosophila willistoni group. Animal Behavior, 83(1), 217-227.

Göpfert, M. C., \& Robert, D. (2003). Motion generation by Drosophila mechanosensory neurons. Proceedings of the National Academy of Sciences, 100(9), 5514-5519.

Greenspan, R. J., \& Ferveur, J. F. (2000). Courtship in Drosophila. Annual Review of Genetics, 34(1), 205-232.

Grossfield, J. (1971). Geographic distribution and light-dependent behavior in Drosophila. Proceedings of the National Academy of Sciences of the United States of America, 68(11), 2669-2673.

Hoffmann, A. A. (1990). The influence of age and experience with conspecifics on territorial behavior in Drosophila melanogaster. Journal of Insect Behavior, 3(1), 1-12.

Holm, S. (1979). A Simple Sequentially Rejective Multiple Test Procedure. Scandinavian Journal of Statistics Scand J Statist, 6(6), 65-70.

Irvin, S. D., Wetterstrand, K. A., Hutter, C. M., \& Aquadro, C. F. (1998). Genetic variation and differentiation at microsatellite loci in Drosophila simulans: Evidence for founder effects in new world populations. Genetics, 150(2), 777-790.

Jallon, J. M. (1984). A few chemical words exchanged by Drosophila during courtship and mating. Behavioral Genetics, 14, 441-478.

Jallon, J.M., \& David, J. R. (1987). Variations in cuticular hydrocarbons among the eight species of the Drosophila melanogaster subgroup. Evolution, 41(2), 294-302.

Kliman, R. M., Andolfatto, P., Coyne, J. A., Depaulis, F., Kreitman, M., Berry, A. J., McCarter, J., Wakeley, J., \& Hey, J. (2000). The population genetics of the origin and divergence of the Drosophila simulans complex species. Genetics, 156(4), 1913-1931.

Kohatsu, S., Koganezawa, M., \& Yamamoto, D. (2011). Female Contact Activates MaleSpecific Interneurons that Trigger Stereotypic Courtship Behavior in Drosophila. Neuron, 69(3), 498-508.

Krstic, D., Boll, W., \& Noll, M. (2009). Sensory integration regulating male courtship behavior in Drosophila. PLoS ONE, 4(2), e4457.

Kyriacou, C. P., \& Hall, J. C. (1982). The function of courtship song rhythms in Drosophila. Animal Behaviour, 30(3), 794-801.

Li, H., \& Stephan, W. (2006). Inferring the Demographic History and Rate of Adaptive Substitution in Drosophila. PLoS Genetics, 2(10), e166. 
513 Lu, B., Zelle, K. M., Seltzer, R., Hefetz, A., Ben-Shahar, Y., Amirav, A., ... Kravitz, E. A.

514

515

516

517

518

519

520

521

522

523

524

525

526

527

528

529

530

531

532

533

534

535

536

537

538

539

540

541

542

543

544

545

546

547

548

549

550

551

552

553

554

555

556

557

558 (2014). Feminization of pheromone-sensing neurons affects mating decisions in Drosophila males. Biology Open, 3(2), 152-60.

Machado, H. E., Bergland, A. O., O’Brien, K. R., Behrman, E. L., Schmidt, P. S., \& Petrov, D. A. (2016). Comparative population genomics of latitudinal variation in Drosophila simulans and Drosophila melanogaster. Molecular Ecology, 25(3), 723-740.

Manning, A. (1959a). The Sexual Behaviour of Two Sibling Drosophila Species. Behaviour, $15(1-2), 123-145$.

Manning, A. (1959b). The sexual isolation between Drosophila melanogaster and Drosophila simulans. Animal Behaviour, 7(1-2), 60-65.

Montell, C. (2009). A taste of the Drosophila gustatory receptors. Current Opinion in Neurobiology, 19(4), 345-353.

Noor, M. A. F., \& Ortiz-Barrientos, D. (2006). Simulating natural conditions in the laboratory: A re-examination of sexual isolation between sympatric and allopatric populations of Drosophila pseudoobscura and D. persimilis. Behavioral Genetic, 36, 322-327.

Pan, Y., Meissner, G. W., \& Baker, B. S. (2012). Joint control of Drosophila male courtship behavior by motion cues and activation of male-speci fi c P1 neurons, 109(25), 1006510070.

Pardy, J. A., Rundle, H. D., Bernards, M. A., \& Moehring, A. J. (2018). The genetic basis of female pheromone differences between Drosophila melanogaster and D. simulans. Heredity, 1.

Pischedda, A., Shahandeh, M. P., Cochrane, W. G., Cochrane, V. A., \& Turner, T. L. (2014). Natural variation in the strength and direction of male mating preferences for female pheromones in Drosophila melanogaster. PLoS ONE, 9(1), e87509.

Roy, P. R., \& Gleason, J. M. (2019). Assessing the use of wing ornamentation and visual display in female choice sexual selection. Behavioral Processes, 158, 89-96.

Salcedo, E., Huber, A., Henrich, S., Chadwell, L. V, Chou, W. H., Paulsen, R., \& Britt, S. G. (1999). Blue- and green-absorbing visual pigments of Drosophila: ectopic expression and physiological characterization of the R8 photoreceptor cell-specific Rh5 and Rh6 rhodopsins. The Journal of Neuroscience: The Official Journal of the Society for Neuroscience, 19(24), 10716-26.

Savarit, F., Sureau, G., Cobb, M. , \& Ferveur, J. F. (1999). Genetic elimination of known pheromones reveals the fundamental chemical bases of mating and isolation in Drosophila. Proceedings of the National Academy of Science. 96, 9015-9020.

Schilcher, F. von. (1976). The function of pulse song and sine song in the courtship of Drosophila melanogaster. Animal Behaviour, 24(3), 622-625.

Seeholzer, L. F., Seppo, M., Stern, D. L., \& Ruta, V. (2018). Evolution of a central neural circuit underlies Drosophila mate preferences. Nature, 559(7715), 564-569.

Shahandeh, M. P., Pischedda, A., \& Turner, T. L. (2018). Male mate choice via cuticular hydrocarbon pheromones drives reproductive isolation between Drosophila species. Evolution, 72(1), 123-135.

Sharma, M. D., Mitchell, C., Hunt, J., Tregenza, T., \& Hosken, D. J. (2012). The Genetics of Cuticular Hydrocarbon Profiles in the Fruit Fly Drosophila simulans. Journal of Heredity, 103(2), 230-239.

Soto-Yéber, L., Soto-Ortiz, J., Godoy, P., \& Godoy-Herrera, R. (2018). The behavior of adult Drosophila in the wild. PLoS One, 13, e0209917. 
559 Spieth, H. T. (1952). Mating behavior within the genus Drosophila (Diptera). Bulletin of the $560 \quad A M N H, 99(7), 395-474$.

561

562

563

564

565

566

567

568

569

570

571

572

573

574

575

576

577

578

579

580

581

582

583

584

Spieth, H. T. (1974). Courtship Behavior in Drosophila. Annual Review of Entomology, 19(1), 385-405.

Spieth, H. T., \& Hsu, T. C. (1950). The Influence of Light on the Mating Behavior of Seven Species of the Drosophila melanogaster Species Group. Evolution, 4(4), 316.

Starostina, E., Liu, T., Vijayan, V., Zheng, Z., Siwicki, K. K., \& Pikielny, C. W. (2012). A Drosophila $\mathrm{DEG} / \mathrm{ENaC}$ subunit functions specifically in gustatory neurons required for male courtship behavior. The Journal of Neuroscience : The Official Journal of the Society for Neuroscience, 32(13), 4665-74.

Stockinger, P., Kvitsiani, D., Rotkopf, S., Tirián, L., \& Dickson, B. J. (2005). Neural circuitry that governs Drosophila male courtship behavior. Cell, 121(5), 795-807.

Thistle, R., Cameron, P., Ghorayshi, A., Dennison, L., \& Scott, K. (2012). Contact chemoreceptors mediate male-male repulsion and male-female attraction during Drosophila courtship. Cell, 149(5), 1140-51.

Toda, H., Zhao, X., \& Dickson, B. J. (2012). The Drosophila female aphrodisiac pheromone activates ppk23(+) sensory neurons to elicit male courtship behavior. Cell Reports, 1(6), 599-607.

Tompkins, L., Gross, A. C., Hall, J. C., Gailey, D. a., \& Siegel, R. W. (1982). The role of female movement in the sexual behavior of Drosophila melanogaster. Behavior Genetics, 12(3), 295-307.

Trajković, J., Miličić, D., Savić, T., \& Pavković-Lučić, S. (2017). Sexual selection, sexual isolation and pheromones in Drosophila melanogaster strains after long-term maintaining on different diets. Behavioral Processes, 140, 81-86.

von Philipsborn, A. C., Liu, T., Yu, J. Y., Masser, C., Bidaye, S. S., \& Dickson, B. J. (2011). Neuronal Control of Drosophila Courtship Song. Neuron, 69(3), 509-522. 


\section{Table $\mathbf{1}$ (on next page)}

Drosophila simulans and D. melanogaster strains.

For each strain we used, the National Drosophila Species Stock Center number is provided. Also provided are the collection location and date (when available), along with the longitude and latitude used to test for correlations of behavior and geography. For strains where a longitude and latitude was not provided by the stock center, we used the longitude and latitude for the collection location. 
1 Table 1: Drosophila simulans and $\boldsymbol{D}$. melanogaster strains. For each strain we used, the

2 National Drosophila Species Stock Center number is provided. Also provided are the collection

3 location and date (when available), along with the longitude and latitude used to test for

4 correlations of behavior and geography. For strains where a longitude and latitude was not

5 provided by the stock center, we used the longitude and latitude for the collection location.

6

\begin{tabular}{|c|c|c|c|c|c|}
\hline Stock \# & Species & $\begin{array}{c}\text { Collection location } \\
\text { (date) }\end{array}$ & Longitude & Latitude & $\begin{array}{c}\text { Strain } \\
\text { label }\end{array}$ \\
\hline $14021-0251.005$ & D. simulans & Lima, Peru (1956) & -77.0428 & -12.0464 & PER005 \\
\hline $14021-0231.01$ & D. melanogaster & Ica, Peru (1956) & -75.7342 & -14.0755 & M-PER01 \\
\hline 14021-0251.009 & D. simulans & $\begin{array}{l}\text { Gorak, New Guinea } \\
\text { (1961) }\end{array}$ & 145.3863 & -6.0835 & NG009 \\
\hline $14021-0231.120$ & D. melanogaster & $\begin{array}{l}\text { Port Moresby, Papua } \\
\text { New Guinea (1982) }\end{array}$ & 147.1803 & -9.4438 & M-NG120 \\
\hline $14021-0251.169$ & D. simulans & South Africa & 22.9375 & -30.5595 & SA169 \\
\hline $\begin{array}{l}14021-0231.51 \\
14021-0231.62\end{array}$ & D. melanogaster & $\begin{array}{l}\text { Cape Town, South } \\
\text { Africa (2007) }\end{array}$ & 18.4241 & -33.9249 & M-SA51 \\
\hline $14021-0251.181$ & D. simulans & $\begin{array}{l}\text { Crete Island, Greece } \\
(2002)\end{array}$ & 24.8093 & 35.2401 & GRE181 \\
\hline $14021-0231.69$ & D. melanogaster & $\begin{array}{l}\text { Athens, Greece } \\
\text { (1965) }\end{array}$ & 23.7275 & 37.9838 & M-GRE69 \\
\hline $14021-0251.261$ & D. simulans & $\begin{array}{l}\text { Lujeri, Malawi } \\
\text { (2009) }\end{array}$ & 35.6484 & -16.0400 & MAL261 \\
\hline $14021-0231.76$ & D. melanogaster & $\begin{array}{l}\text { Lujeri, Malawi } \\
\text { (2009) }\end{array}$ & 35.6484 & -16.04 & M-MAL76 \\
\hline $14021-0251.288$ & D. simulans & $\begin{array}{l}\text { Athens, Georgia } \\
(2009)\end{array}$ & 83.3576 & 33.9519 & GEO288 \\
\hline $14021-0231.183$ & D. melanogaster & $\begin{array}{l}\text { Athens, Georgia } \\
\text { (2009) }\end{array}$ & 83.3576 & 33.9519 & $\begin{array}{c}\text { M- } \\
\text { GEO181 }\end{array}$ \\
\hline $14021-0251.004$ & D. simulans & Australia (1955) & 133.7751 & -25.2744 & AUS004 \\
\hline $14021-0231.03$ & D. melanogaster & $\begin{array}{l}\text { Queensland, } \\
\text { Australia }\end{array}$ & 142.7028 & -20.9176 & M-AUS05 \\
\hline $14021-0251.166$ & D. simulans & IslaMorada, Florida & -80.6278 & 24.9243 & FLO166 \\
\hline 14021-0231.14 & D. melanogaster & Orlando, Florida & -81.3792 & 28.5383 & M-FLO14 \\
\hline $14021-0251.001$ & D. simulans & $\begin{array}{l}\text { Georgetown, Guyana } \\
\text { (1956) }\end{array}$ & -58.1551 & 6.8013 & GUY001 \\
\hline $14021-0231.15$ & D. melanogaster & Bahia, Brazil & -41.7007 & -12.5797 & $\begin{array}{c}\text { M- } \\
\text { BRAZ15 }\end{array}$ \\
\hline 14021-0251.196 & D. simulans & $\begin{array}{l}\text { Ansirabe, } \\
\text { Madagascar (1998) }\end{array}$ & 47.0291 & -19.873 & MAD196 \\
\hline $14021-0231.125$ & D. melanogaster & $\begin{array}{l}\text { Tananarive, } \\
\text { Madagascar (1982) }\end{array}$ & 47.5079 & -18.8792 & $\begin{array}{c}\text { M- } \\
\text { MAD125 }\end{array}$ \\
\hline 14021-0251.006 & D. simulans & $\begin{array}{l}\text { Nueva, California } \\
\text { (1961) }\end{array}$ & -117.1459 & 33.8014 & NUE006 \\
\hline 14021-0231.131 & D. melanogaster & $\begin{array}{l}\text { La Jolla, California } \\
\text { (2009) }\end{array}$ & -117.2713 & 32.8328 & M-SD131 \\
\hline
\end{tabular}




\section{Figure 1}

Copulation success of $11 \mathrm{D}$. simulans strains by treatment and time point.

A. The copulation success of pairs held for 2 hours in the light (yellow circles) compared to in the dark (blue squares). B. The copulation success of pairs held for 6 hours in the light (yellow circles) compared to in the dark (blue squares). C. The relative light independence of copulation success in the dark compared to in the light is shown for each strain for 2 hours (light grey squares) and 6 hours (black circles). For all, paired data points between treatments are connected with a line. Corresponding points are labelled with their strain label (Table 1). Individual strains and significance values after correction for multiple comparisons are indicated to the right of each point. Species wide differences are indicated with asterisks above plots $\left(*=p<0.05,{ }^{* * *}=p<0.001\right.$, and $\left.{ }^{* * * *}=p<0.0001\right)$.

A.

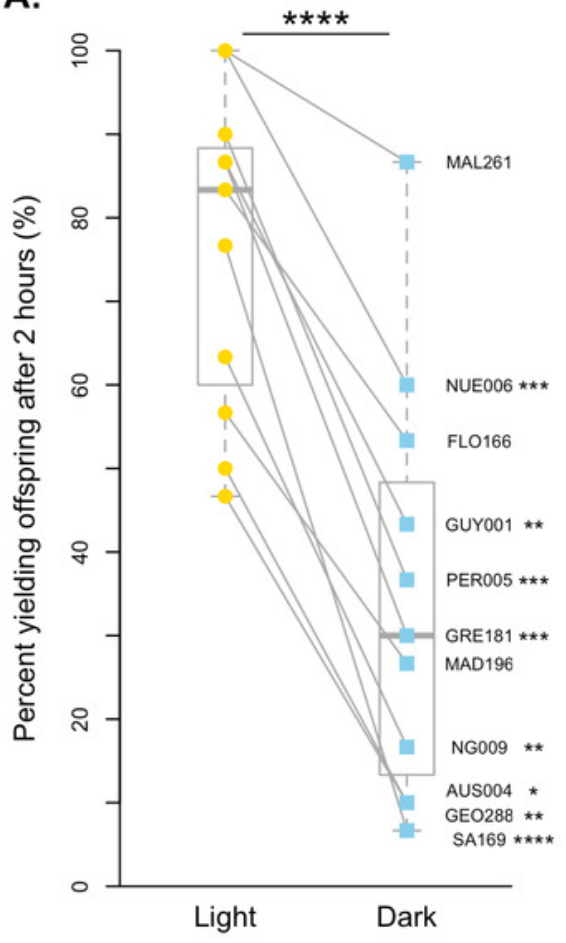

B.

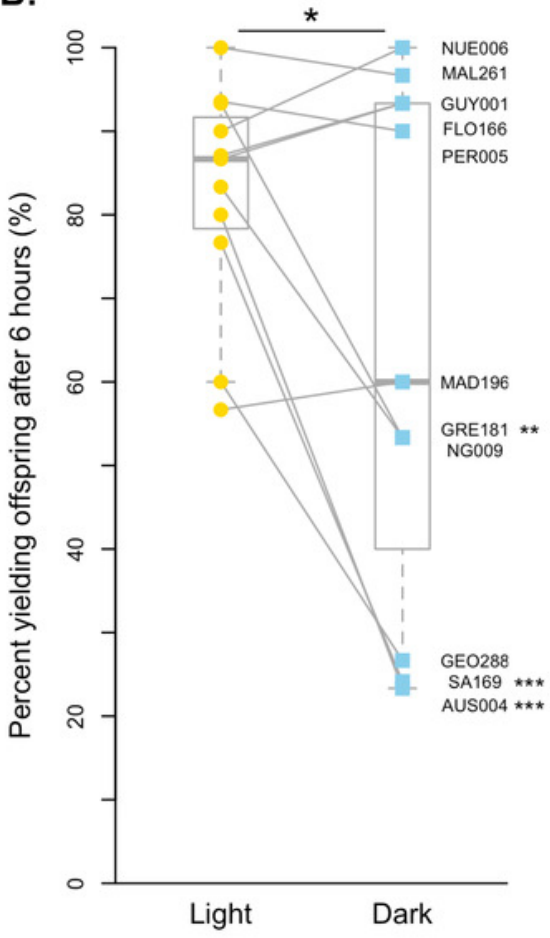

C.

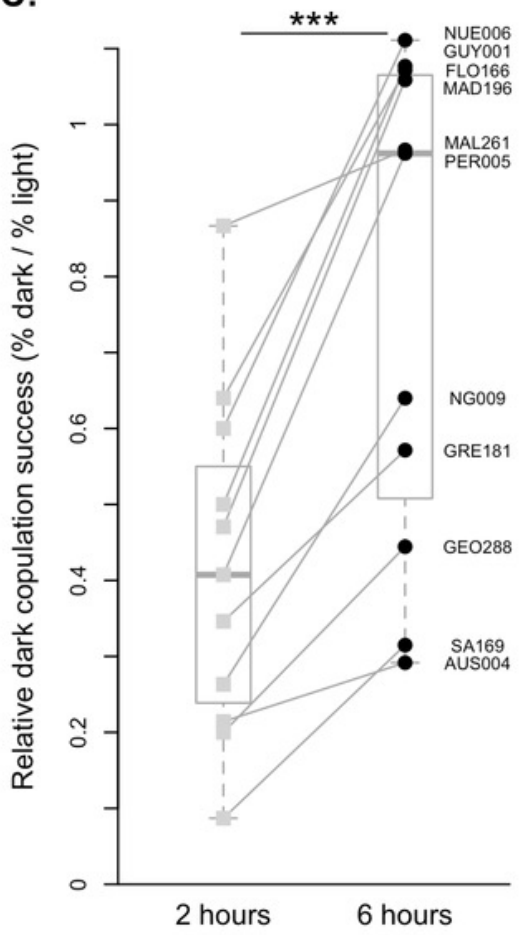




\section{Figure 2}

Copulation success of $11 \mathrm{D}$. melanogaster strains by treatment and time point.

A. The copulation success of pairs held for 2 hours in the light (yellow circles) compared to in the dark (blue squares). B. The copulation success of pairs held for 6 hours in the light (yellow circles) compared to in the dark (blue squares). C. The relative light independence of copulation success in the dark compared to in the light is shown for each strain for 2 hours (light grey squares) and 6 hours (dark grey circles). For all, paired data points between treatments are connected with a line. Corresponding points are labelled with their strain label (Table 1). Individual strains and significance values after correction for multiple comparisons are indicated to the right of each point. Species wide differences are indicated with asterisks above plots $\left(* *=p<0.01\right.$ and $\left.{ }^{* * *}=p<0.001\right)$.

A.

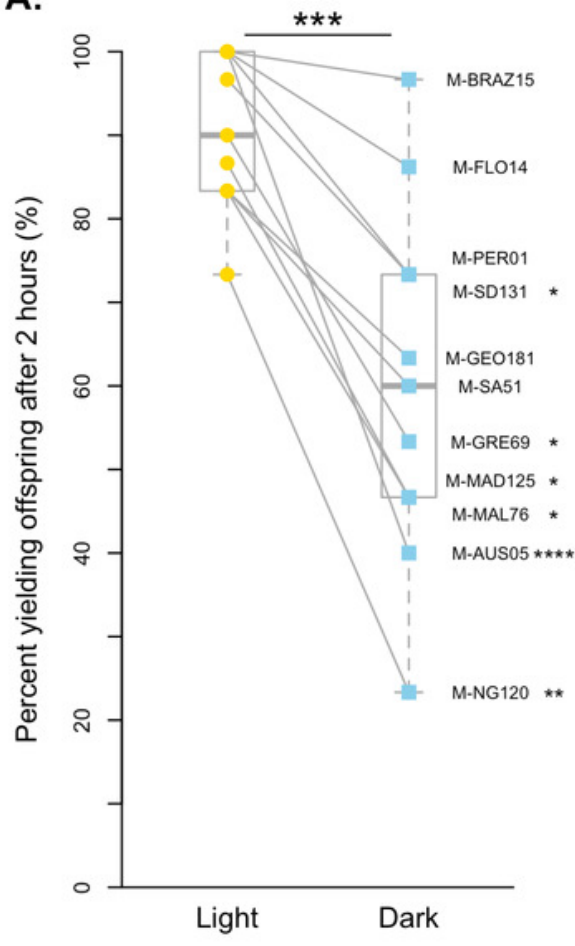

B.

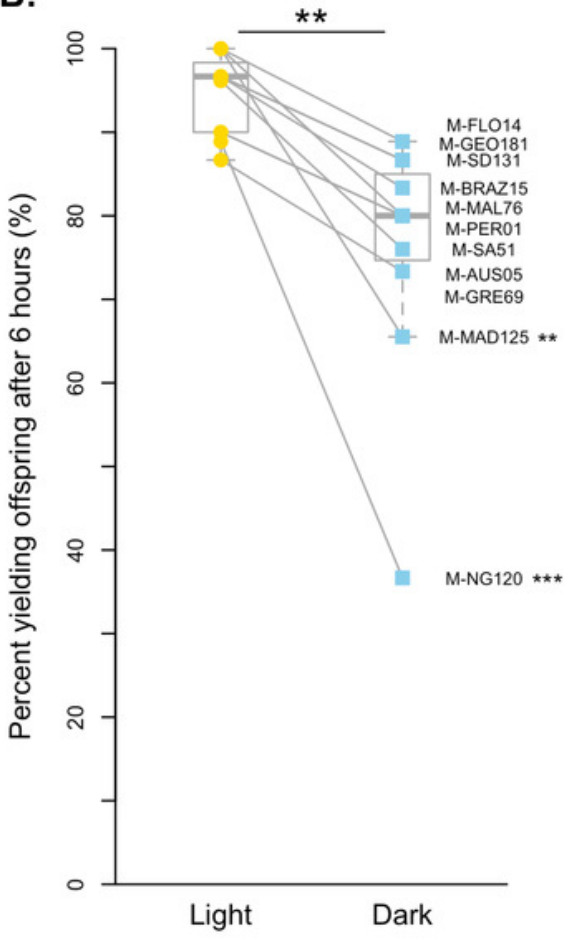

C.

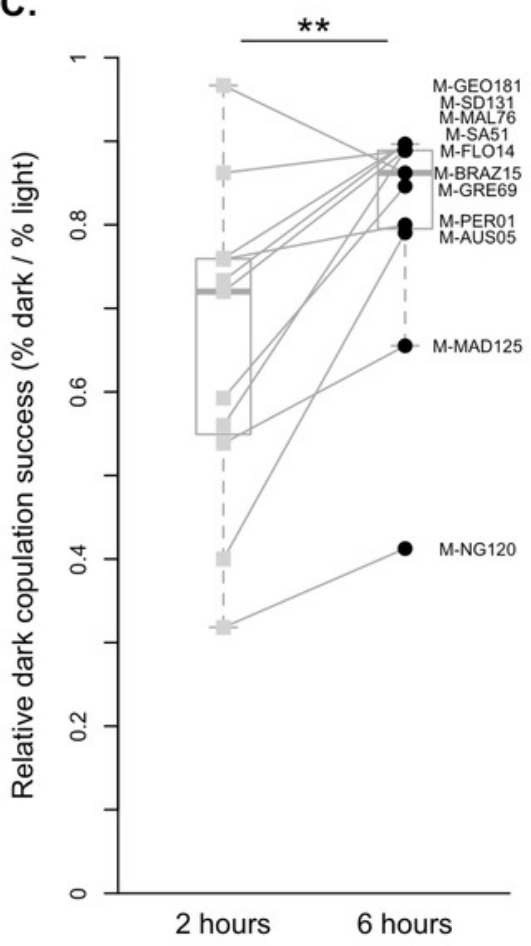




\section{Figure 3}

Figure 3. Differences in dark courtship ability correlate with longitude in $D$. melanogaster but not $D$. simulans.

A. The relative dark copulation success at 2 hours is plotted by geographic sample for $D$. simulans strains (blue) and D. melanogaster strain (yellow). B. There is no correlation between relative light dependence at 2 hours ( $y$-axis) and longitude ( $\mathrm{x}$-axis) for $D$. simulans

strains. C. There is a significant correlation between relative light dependence at 2 hours ( $y$ axis) and longitude (x-axis) for $D$. melanogaster strains. For $B$ and $C$, individual points are labelled with their strain label (Table 1). The red dashed line represents the best fit line from a linear model Pearson's correlation coefficient and significance values, corrected for multiple comparisons, are displayed in the upper left corner of the plot. 

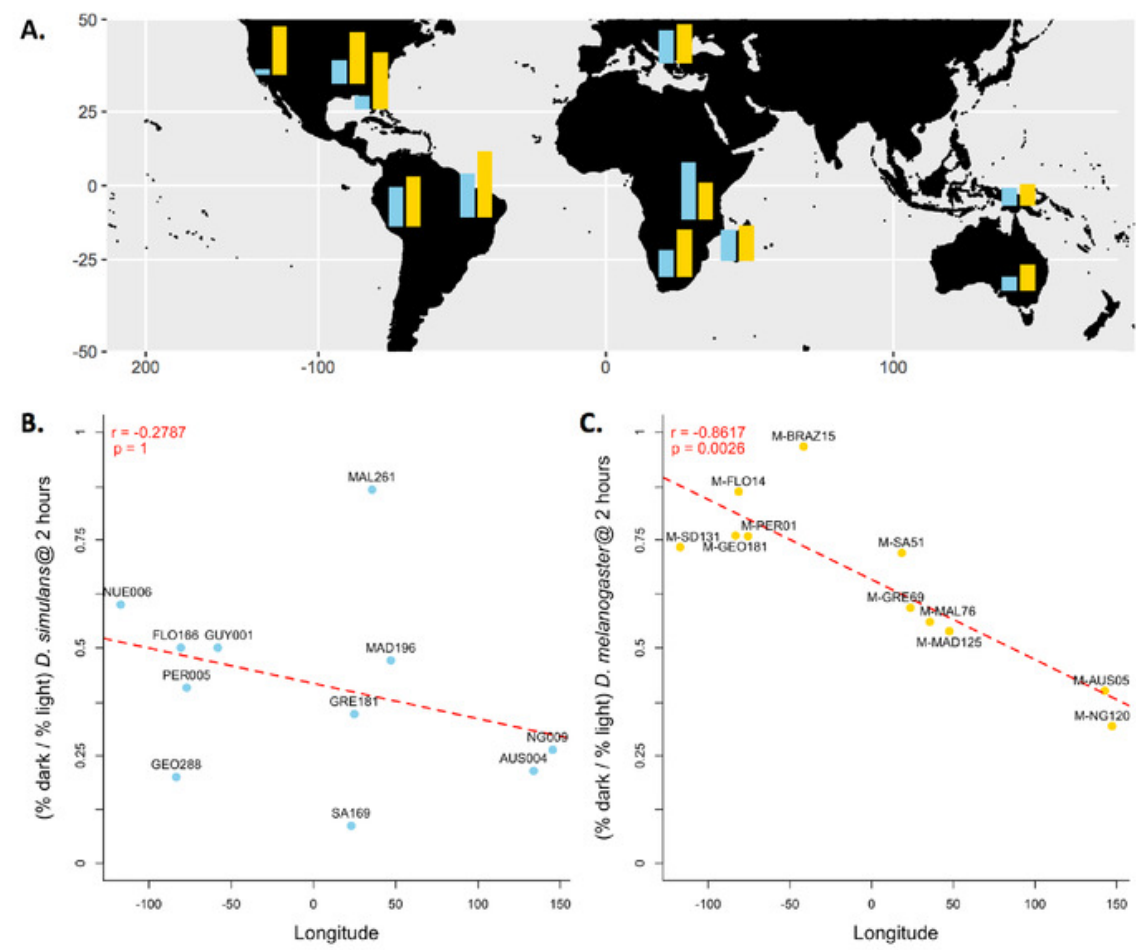


\section{Figure 4}

Courtship behavior of light dependent and light independent $D$. melanogaster and $D$. simulans under bright light and in darkness.

A. The percent of $D$. melanogaster strain males that court their own females in the light (yellow bars) compared to in darkness (blue bars). M-BRAZ15 was the most light-independent strain from our copulation assay, while M-NG120 was the most light-dependent ( $\mathrm{N}=10$ for both). B. The percent of $D$. simulans strain males that court their own females in the light (yellow bars) compared to in darkness (blue bars). $\mathrm{N}=4$ in the light, and $\mathrm{N}=7$ in the dark for both strains. C. The percent of $D$. simulans strain males that court the opposing strain's females in the light (yellow bars) compared to in darkness (blue bars). $\mathrm{N}=3$ for all except for MAL261 males with SA169 females in the dark, where $N=4$. For B and C, MAL261 was the most light-independent strain from our copulation assay, while SA169 was the most lightdependent. $*=p<0.05$ 
A.

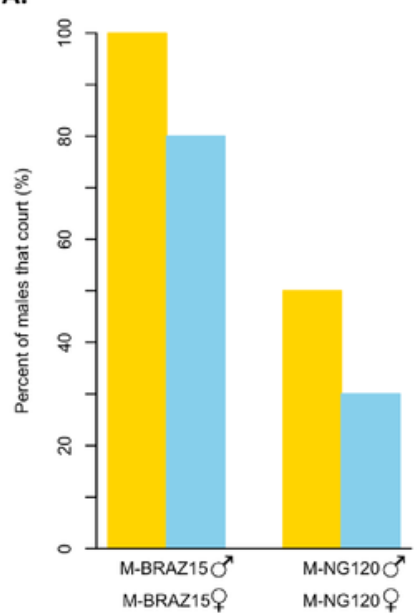

B.

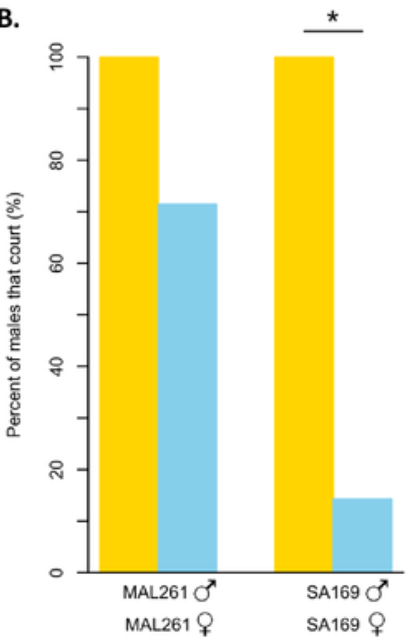

C.

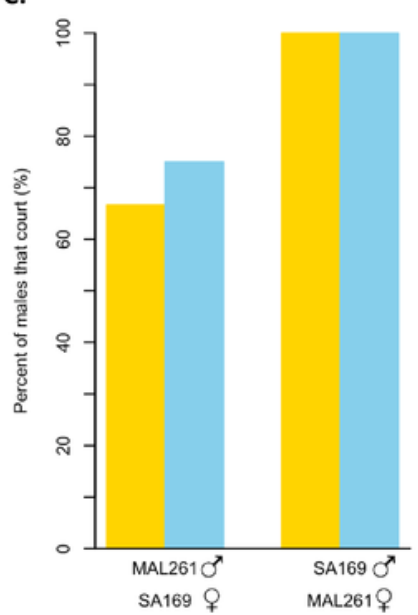

